Article

\title{
ERP Indices of Stimulus Prediction in Letter Sequences
}

\author{
Edith Kaan * and Evan Carlisle \\ Department of Linguistics, University of Florida, Box 115454, Gainesville, FL 32611, USA; \\ E-Mail: e.carlisle1017@gmail.com \\ * Author to whom correspondence should be addressed; E-Mail: kaan@ufl.edu; \\ Tel.: +1-352-392-0639; Fax: +1-352-392-8480.
}

External Editor: Germán Barrionuevo

Received: 26 May 2014; in revised form: 25 August 2014 / Accepted: 22 September 2014 /

Published: 23 October 2014

\begin{abstract}
Given the current focus on anticipation in perception, action and cognition, including language processing, there is a need for a method to tap into predictive processing in situations in which cue and feedback stimuli are not explicitly marked as such. To this aim, event related potentials (ERPs) were obtained while participants viewed alphabetic letter sequences ("A", "B", "C", "D”, “E”, ...), in which the letters were highly predictable, and random sequences ("S", "B", “A”, "I", "F”, “M", ...), without feedback. Occasionally, the presentation of a letter in a sequence was delayed by $300 \mathrm{~ms}$. During this delay period, an increased negativity was observed for predictive versus random sequences. In addition, the early positivity following the delay was larger for predictive compared with random sequences. These results suggest that expectation-sensitive ERP modulations can be elicited in anticipation of stimuli that are not explicit targets, rewards, feedback or instructions, and that a delay can strengthen the prediction for a particular stimulus. Applications to language processing will be discussed.
\end{abstract}

Keywords: predictive processing; anticipatory processing; P2; P3b; stimulus preceding negativity; SPN; omission error; MMN; contingent negative variation; $\mathrm{CNV}$

\section{Introduction}

The idea that cognition and perception are anticipatory in nature has recently regained scholarly attention [1,2]. Humans continuously make predictions concerning upcoming events or stimuli and 
match these predictions to the actual input. When expectations are not met, the resulting error signal is used to extract important information and adjust expectations. The ability to predict therefore underlies the ability to learn and adapt. Predictive processing is an essential part of perception, action and cognition [1,2], including language processing [3-10]. Therefore, a growing need exists for experimental techniques and designs that can tap into predictive processing. Event-related-potentials (ERPs) are an appropriate method, since this technique enables the continuous recording of brain activation during cognitive processing.

One class of ERP components associated with anticipatory processing are stimulus preceding negativities (SPNs) [11]. SPNs are a collection of negativities whose scalp distribution varies depending on the task and type of information that is expected [12,13]. SPNs are typically elicited in delay paradigms. In these paradigms, a particular stimulus is temporally separated from a subsequent stimulus, which can occur with a varying degree of predictability. SPNs have typically been observed in the period: before a stimulus that conveys feedback on the performance of the preceding stimulus; before a stimulus that conveys instructions; before a probe to which previous stimuli or task outcomes need to be compared; or before a stimulus that is expected to be associated with a strong emotion [13]. The SPN is not an index of expectancy of stimuli in general, however. First, not all stimuli that are highly predictable give rise to an SPN. For instance, the expected occurrence of an instruction stimulus at a particular point in a trial does not [14]. Second, the SPN can be larger when the nature of the following stimulus is less, rather than more, predictable [15-17]. One interpretation is that the SPN is larger when the participant expects the upcoming stimulus to be more emotionally informative [16,18].

Another ERP response associated with anticipation is an omission-related negativity, also regarded as a mismatch negativity (MMN) and/or an N2 component [19-21]. This component has been observed in auditory paradigms in which an expected stimulus is occasionally either briefly delayed or omitted and replaced with a brief period of silence. The omission-related negativity can be modulated by the degree of expectation: Its amplitude is larger when the omitted stimulus is more strongly expected [22,23]. Omission-related effects have also been reported in studies using visual presentation in which the temporal regularity of stimulus presentation is occasionally disrupted [24,25]; to our knowledge, however, no omission studies have manipulated the predictability of the upcoming stimulus in a visual paradigm.

The present study aimed to test whether anticipation-related effects could be elicited in a delay paradigm in which pre-learned visual sequences were used and no explicit feedback was provided. Participants were presented with letter sequences, which could be either in alphabetical order, in which the next letter was $100 \%$ predictable, or in a random order, in which the next letter was about $5 \%$ predictable. In both types of sequence, half of the trials had a lengthened inter stimulus interval (ISI) between two of the letters, creating a delay. The task was an end-of-trial letter recognition task, which was unrelated to the predictability of the sequence or the presence of a delay. If participants form predictions of the next letter on the basis of the preceding letters, the delay would either strengthen the expectation of the next letter or, alternatively, violate the expectation of the presentation of a particular letter at a certain point in time. In either case, we expected differences in amplitude between the predictable and random sequences.

In addition, we were interested in the effect of predictability and delay on the degree to which the next letter was pre-activated. One interpretation of anticipatory processing is that the perception of 
upcoming stimuli is facilitated by the pre-activation of the relevant sensory and cognitive systems. This facilitation may be reflected in the modulation of early sensory ERP components when the expected stimulus occurs. The P2 is a component typically occurring between 100 and $300 \mathrm{~ms}$. The P2 has been associated with early perceptual processes, which can be modulated by top-down information. In the visual domain, a larger P2 amplitude has been associated with the ease in which relevant (pre-specified, target-related) visual features, such as a particular color or size, can be detected [26]. In the language domain, the P2 is larger for repeated words [27,28] and larger for plausible words in a highly-constraining versus a weakly-constraining semantic context [29,30]. Evans and Federmeier [27] (p. 305) suggest that, since the P2 is sensitive to word repetition, "it may reflect the process of comparing visual input with either stored knowledge or generated expectations". In addition, the P300, and in particular the P3b, has been found to be larger for stimuli that are highly expected in a context and has been interpreted as indexing the matching of expectation, that is, the match of the actual stimulus with a pre-activated item [31-33].

We therefore expected a larger P2 and/or P3b for elements in predictive versus non-predictive sequences. If a lengthened ISI makes the prediction stronger and leads to more pre-activation of the anticipated element, we expected a larger P2/P3b after a delay than without a delay, especially for predictive sequences.

\section{Methods}

\subsection{Participants}

Nineteen native speakers of American English were recruited from the University of Florida community. Data from one participant were excluded because of chance performance on the end-of-trial probe verification task, leaving a total of 18 participants (age 18-30, mean 20.9; 4 men). All, except for one, participants were right-handed. All had normal or corrected-to-normal vision, had no reading problems and were neurologically healthy according to a self-report. All participants were able to generate the letters of the Roman alphabet in the correct order when asked before the study. Participants were either paid $\$ 10.00$ per hour or received course credit for their participation. All participants gave written informed consent as per the University of Florida Institutional Review Board procedures (Protocol \#2013-U-0768, approved 6/19/2013).

\subsection{Materials}

Stimuli consisted of 180 sequences of upper-case letters of the Roman alphabet. First, 45 predictive, alphabetical sequences (e.g., "E", "F", “G”, "H”, "I", “J”, "K”) were formed. Sequences were between 7 and 10 letters long. Random sequences were derived from the predictive sequences, such that the random and predictive sequences did not differ in the number and distribution of particular letters over the positions in the sequence. When creating random sequences, strings of letters were avoided that may form known acronyms or other recognizable patterns (e.g., "OMG", "LOL", etc.). From these 90 no-delay sequences, 90 delay trials were created by adding a delay (that is, a lengthened ISI) after the fourth to eighth letter (nine instances for each of these five positions for the predictive and random conditions). Care was taken that the position of the delay in the predictive sequences did not occur in a 
place where a pause may feel natural due to the pauses in the "ABC Song" (after "G", "P", "S", "V", and "X") and that the delay was never in a pre-final position. This was to prevent participants from expecting a delay in a certain position, and to encourage them to keep processing the stimuli even after the delay. Each participant saw all 180 trials (45 for each of the 4 conditions: predictive delay; random delay; predictive no delay; random no delay). Each of the trials was followed by a letter prompt. In half of the trials, equally distributed across conditions, the prompt letter was a member of the sequence ("yes" trials); in the other half, it was not ("no" trials). Prompt letters in the "yes" trials were equally drawn from the various letter positions, with the position balanced equally over the random and predictive sequences.

\subsection{Procedure}

Participants were seated comfortably in a sound-attenuating room, $1 \mathrm{~m}$ away from an SVGA monitor. Letters were presented in Courier New 24 point font, white letters on a black background. Each sequence began with a fixation presented in the center of the screen for $1000 \mathrm{~ms}$, followed by a blank screen of $200 \mathrm{~ms}$. Letters were presented individually for $300 \mathrm{~ms}$ followed by a blank screen (ISI) of $200 \mathrm{~ms}$. In the conditions containing a delay, one of the letters in Positions 5 through 9 was followed by an ISI of $500 \mathrm{~ms}$ rather than $200 \mathrm{~ms}$, thus creating a delay of $300 \mathrm{~ms}$ relative to the expected onset time. The final letter of each sequence was presented with a period. After a blank screen of $1000 \mathrm{~ms}$, a prompt letter was presented. At this point, participants indicated whether or not that letter had occurred in the preceding sequence by pressing the left ("yes") or right ("no") trigger button on a game pad. After responding to the prompt, the message "Press to continue" remained on the screen until the participant pressed a button on the gamepad to proceed to the next trial.

Participants were instructed to limit movements and blinking to the pauses at the end of each sequence (during the prompt or after). Upon the completion of a practice block, which consisted of six sequences (three alphabetic, three random, none containing a disruption), participants completed three blocks, each consisting of 60 sequences. Each block contained 15-16 trials from each of the four conditions, in a randomized order. The order of the three blocks was varied such that no more than two participants saw the blocks in a given order.

\subsection{EEG Recording}

EEG was recorded with $64 \mathrm{Ag} / \mathrm{AgCl}$ electrodes mounted in an elastic cap (Waveguard 64). The following electrode positions were used: on the midline (FPz, Fz, FCz, Cz, CPz, Pz, POz, Pz) and lateral sites (FP1/2, AF3/4/7/8, F1-8, FC1-6, FT7/8, C1-6, T7/8, CP1-6, TP7/8, P1-8, PO3-8, O1/2). Horizontal EOG was recorded with two $\mathrm{Ag} / \mathrm{AgCl}$ electrodes attached to the left and right outer canthi of both eyes, and vertical $\mathrm{EOG}$ was recorded with $\mathrm{Ag} / \mathrm{AgCl}$ electrodes above and below the right eye. Additional electrodes were placed on the left and right mastoids. Impedances never exceeded $5 \mathrm{k} \Omega$ at all times for all electrodes. EEG was acquired using an ANT REFA 8-72 amplifier, at a sampling rate of 512 $\mathrm{Hz}$, referenced to the left mastoid. 


\subsection{Analysis}

Data were filtered offline using a $0.01-30 \mathrm{~Hz}$ band pass filter and arithmetically re-referenced to the mean of both mastoids. Trials with excessive eye movements (standard deviation exceeding $20 \mu \mathrm{V}$ for horizontal EOG; greater than 30 for vertical EOG in a 200-ms window) and trials in which one of the channels was malfunctioning as determined by visual inspection were rejected from analysis. The rejection rate was equally distributed among the four conditions (predictive, delay 16.5\%; predictive, no delay, $16.5 \%$; random, delay $16.4 \%$; random, no delay $16.2 \%$ ). ERPs were time-locked to the onset of the presentation of the letter preceding the potential delay. Epochs spanned $2000 \mathrm{~ms}$, from -500 to $1500 \mathrm{~ms}$, relative to stimulus onset. As will be explained below, the 200-ms time window immediately preceding the pre-delay letter may have been confounded by pre-existing differences between the conditions. For this reason, we report an analysis using the -500 to -300 -ms interval preceding the onset of the pre-delay letter as the baseline. This window coincided with the first $200 \mathrm{~ms}$ after onset of the second letter preceding the potential delay. To investigate the effects of random versus predictive sequences, without contamination of the nature or the preceding stimuli or presence of a delay, we also investigated the ERP for the first three letters in the trial, using the 100-ms interval preceding the onset as a baseline (a longer baseline was too contaminated with eye movements spilling over from the preceding fixation). Epochs were $1600 \mathrm{~ms}$ in length, from -100 to $1500 \mathrm{~ms}$ from the onset of the first letter.

Statistical analyses were performed on the mean of five of the midline electrodes $(\mathrm{Fz}, \mathrm{FCz}, \mathrm{Cz}, \mathrm{CPz}$, $\mathrm{Pz}$ ) and 30 lateral electrodes grouped into 10 regions (left frontal: F1/3/5; right frontal: F2/4/6; left fronto-central: $\mathrm{FC} 1 / 3 / 5$; right fronto-central: $\mathrm{FC} 2 / 4 / 6$; left central: $\mathrm{C} 2 / 4 / 6$; right central: $\mathrm{C} 1 / 3 / 5$; left centro-parietal: CP2/4/6; right centro-parietal: $\mathrm{CP} 1 / 3 / 5$; left parietal: $\mathrm{P} 2 / 4 / 6$; and right parietal: $\mathrm{P} 1 / 3 / 5$ ). Critical time windows were: (1) -300 to $0 \mathrm{~ms}, 0-300 \mathrm{~ms}$ and $300-500 \mathrm{~ms}$ relative to the onset of the pre-delay letter; this was to examine potential differences between the conditions before the onset of the delay; (2) 600 to $900 \mathrm{~ms}$ after onset of the pre-delay letter and corresponding letter in the no-delay conditions; this interval captured the delay-related ERPs; (3) the P2/P3b, which was defined as the 100-ms window surrounding the peak latency averaged over midline sites. This corresponded to the 680-780-ms window from the start of the pre-delay letter for the no-delay conditions and the 980-1080-ms window for the delay conditions. The critical time windows for the analysis of the first three letters of each trial were: (1) 690-780-ms window from the onset of the first letter, capturing the P2/P3 to the second letter; (2) the mean amplitude between 800 and $1000 \mathrm{~ms}$; and (3) 1300-1500 ms after the onset of the first letter, based on visual inspection. SPSS GLM repeated measures analyses were performed on the mean amplitude in the time windows defined, separately for midline and lateral sites. Within-subject factors were order (2 levels: predictive/random), delay (2 levels: delay/no delay), when applicable, and anteriority (5 levels: frontal, fronto-central, central, centro-parietal, parietal). Lateral analyses were performed with the addition of hemisphere (2 levels: left/right) as a factor. Significant interactions involving the factors delay or order were followed up with ANOVAs on the relevant subset of the data. For effects involving more than one degree of freedom, the Greenhouse-Geisser correction was applied to protect against Type I error due to a violation of sphericity [34].

Accuracy for the end-of-trial probe verification task and response times to accurate responses were analyzed with an SPSS GLM repeated measures with order (2) and delay (2) as within-subjects factors. 
Outliers in reaction times were omitted when they exceeded the median plus 3-times the median absolute deviation [35]. This affected $8.3 \%$ of all data points.

\section{Results and Discussion}

\subsection{Behavioral Data}

The mean proportion of accurate responses and response times for accurate responses in the end-of-trial letter recognition task are given in Table 1. Participants were very accurate in indicating whether the post-trial prompt letter was among the letters seen in the trial. Responses were more accurate, but took longer, in the predictive than in the random conditions (accuracy, $F(1,17)=23.85$, $p<0.001$; response times, $F(1,17)=23.46, p<0.001)$, suggesting a speed/accuracy trade-off. Responses were more accurate $(F(1,17)=4.81, p<0.05)$ and faster $(F(1,17)=11.16, p<0.05)$ when the sequence did not contain a delay, suggesting that the presence of a delay hindered the encoding or retrieval of the sequence.

Table 1. Mean proportion of accurate responses and mean response times (RT) in ms to accurate responses in the letter recognition task (standard deviation in parentheses).

\begin{tabular}{ccc}
\hline Condition & Mean Accuracy (SD) & Mean RT (SD) \\
\hline Predictive, delay & $0.94(0.04)$ & $1225(231)$ \\
Random, delay & $0.88(0.06)$ & $1153(209)$ \\
Predictive, no delay & $0.96(0.04)$ & $1195(232)$ \\
Random, no delay & $0.89(0.06)$ & $1099(216)$ \\
\hline
\end{tabular}

\subsection{ERPs: Delay-Related Effects (Time Locked to Pre-Delay Letter)}

Figure 1 displays the ERPs time-locked to the onset of the pre-delay letter in the delay conditions; Figure 2 displays the ERPs for the corresponding position in the no-delay conditions. The delay occurred $500-800 \mathrm{~ms}$ after the onset of the pre-delay letter. Shortly after the onset of the delay, a negative going potential was seen (600-900 ms, gray vertical bars in Figure 1), which was larger for the predictive than the random sequences. In addition, letters following the delay elicited a larger positivity between 180 and $280 \mathrm{~ms}$ after onset than letters in comparable positions without a delay (dotted interval in Figures 1 and 2). This positivity was larger in amplitude for the predictive than for the random sequences, but only after a delay. Superimposed on this effect was a frontal negativity for the random versus the predictive delay conditions, which lasted until the end of the epoch. Note that the random and predictive conditions differed right before the onset of the pre-delay letter, -300 to $0 \mathrm{~ms}$ in Figures 1 and 2 . In this time-window, the ERPs in the random conditions were more negative than in the predictive conditions, which is the reverse pattern of that observed during the delay period. 
Figure 1. Event-related potentials (ERPs) time-locked to the onset of the pre-delay letter in the delay conditions for 25 selected electrodes (left hemisphere (F3, F5, FC3, FC5, C3, C5, CP3, CP5, P3, P5); midline (Fz, FCz, Cz, CPz, Pz); right hemisphere (F4, F6, FC4, FC6, C4, $\mathrm{C} 6, \mathrm{CP} 4, \mathrm{CP} 6, \mathrm{P} 4, \mathrm{P} 6)$. The delay period $(500-800 \mathrm{~ms})$ is indicated with the black bar above the time scale; the letter following the delay starts at $800 \mathrm{~ms}$. Blue line: predictive (alphabetic) condition; red line: random condition. Gray vertical bar: 600-900-ms interval used for the analysis of the effects during the delay; vertical dotted lines: analysis window for the P2/P3 effects. ERPs are baselined to the $200 \mathrm{~ms}$ following the onset of the second letter before the potential delay ( -500 to $-300 \mathrm{~ms}$, see the green bar above the time scale). In this and the following figures, negative is plotted up.

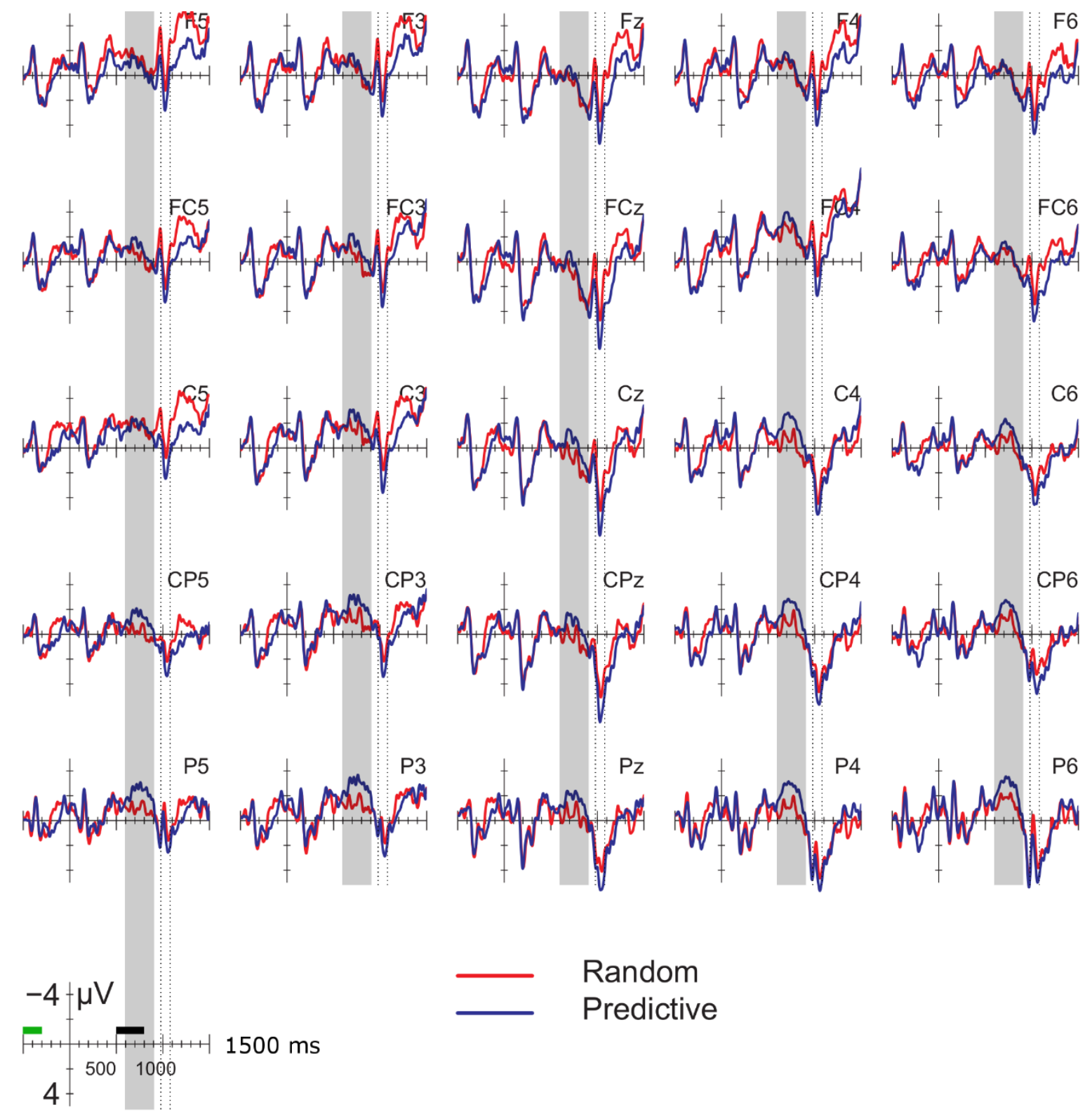


Figure 2. ERPs for the no-delay conditions, time-locked to the onset of the letter equivalent in position to the pre-delay letter in Figure 1. The next letter starts at $500 \mathrm{~ms}$. Blue line: predictive (alphabetic) condition; red line: random condition. ERPs are baselined to the -500 to $-300 \mathrm{~ms}$ interval (green bar). Gray vertical bar: 600-900 ms interval used in the analysis of the delay effects; vertical dotted lines: analysis window for the P2/P3 effects.

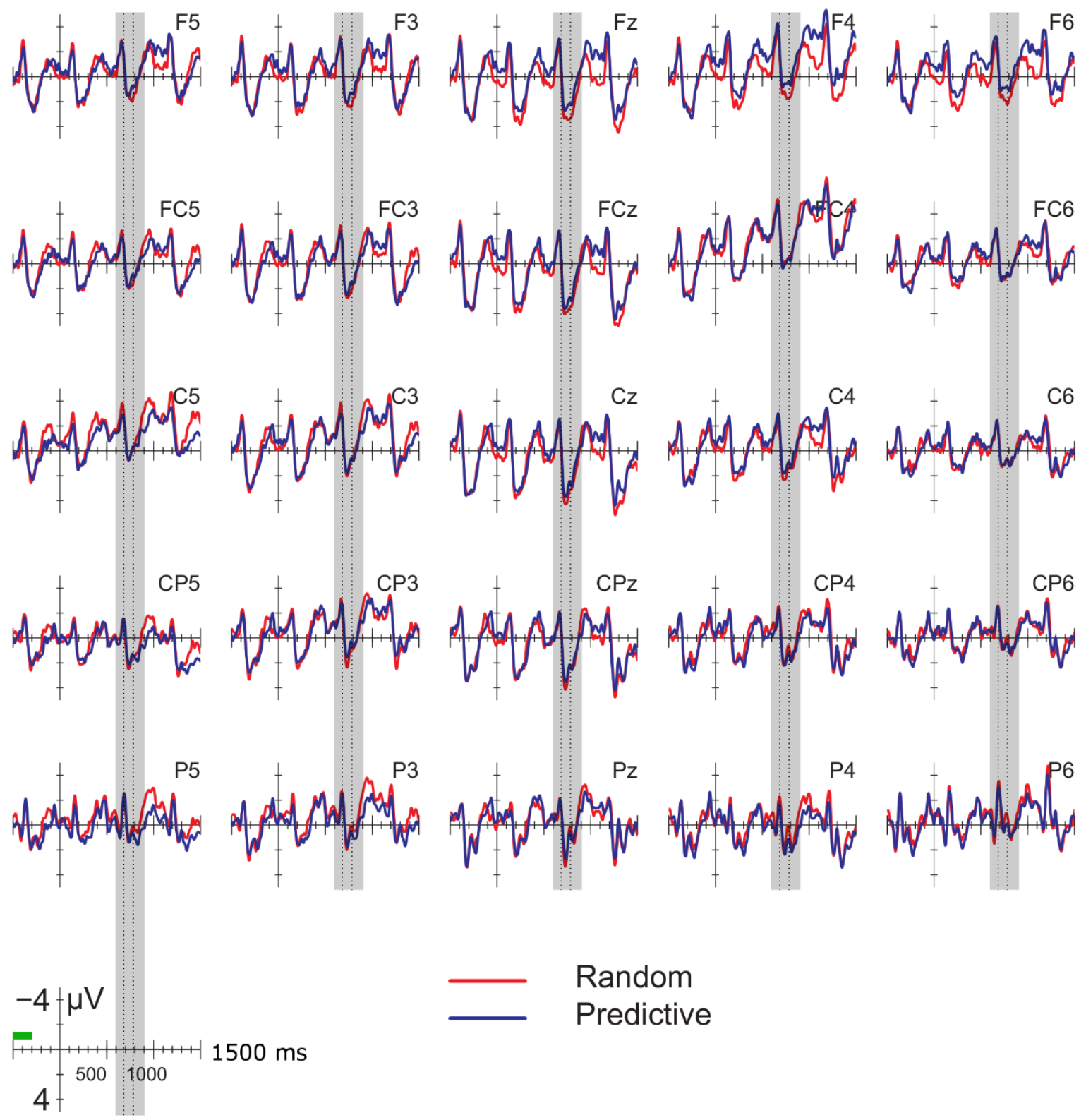

\subsubsection{Pre-Delay Period}

In order to assess potential differences in ERPs between the conditions before the delay, we conducted analyses on the 300-ms time window preceding the pre-delay letter, and on the 0-300-ms and 300-500-ms time windows from the onset of the pre-delay letter.

In the 300-ms interval preceding the pre-delay letter, the ERPs in the random conditions were more negative than in the predictive conditions at lateral sites, which just failed to reach significance $(F(1,17)=4.352, p=0.052)$. This is the opposite pattern of that found in the delay period, where the ERPs to the predictive condition were more negative than the random. No other significant effects were 
obtained in the -300 to 0 -ms or $0-300-\mathrm{ms}$ intervals. In the $300-500-\mathrm{ms}$ time window, no differences were seen among the conditions except for a significant interaction of delay, anteriority and hemisphere at lateral sites $(F(4,68)=5.635, p<0.01)$. This effect could be attributed to the ERPs being more negative in the no-delay than delay conditions over left posterior sites. The direction of this delay effect is opposite of that found in the analysis of the delay interval, during which the ERPs in the delay conditions were more negative. The differences observed in later intervals therefore cannot be ascribed to differences seen before the delay.

\subsubsection{Delay Period}

ERPs shortly after the onset of the delay period (see Figure 1) were more negative over posterior sites compared to when there was no delay (Figure 2). This delay-related negativity was larger for the predictive than the random sequences, whereas there were no differences between the random and predictive order in the no-delay conditions. These observations were confirmed by statistical analyses of the mean in the 600-900-ms interval after the onset of the pre-delay letter. Results from the ANOVA are presented in Table 2. Analyses showed a robust interaction of delay and anteriority. Separate analyses for each of the anterior-posterior sites (Table 3) confirmed that the delay-related negativity was most prominent at posterior sites.

Table 2. Results from ANOVA ( $F$-values) on the mean amplitudes in the 600-900-ms interval from the onset of the pre-delay letter.

\begin{tabular}{ccc}
\hline Effect (df) & Midline & Lateral \\
\hline Order $(1,17)$ & 2.037 & 1.531 \\
Delay $(1,17)$ & $4.094^{+}$ & $11.678^{* *}$ \\
Order $\times$ Anteriority $(4,68)$ & 0.246 & 0.155 \\
Delay $\times$ Anteriority $(4,68)$ & $6.717^{* *}$ & $9.566^{* *}$ \\
Order $\times$ Hemi $(1,17)$ & - & 0.564 \\
Delay $\times$ Hemi $(1,17)$ & - & 0.820 \\
Order $\times$ Anteriority $\times$ Hemi $(4,68)$ & - & 1.317 \\
Delay $\times$ Anteriority $\times$ Hemi $(4,68)$ & - & $3.451^{*}$ \\
Order $\times$ Delay $(1,17)$ & 0.951 & $4.154^{+}$ \\
Order $\times$Delay $\times$Anteriority $(4,68)$ & 2.052 & $3.418^{+}$ \\
Order $\times$Delay $\times$Hemi $(1,17)$ & - & 0.009 \\
Order $\times$ Delay $\times$ Anteriority $\times$ Hemi $(4,68)$ & - & 0.170 \\
Anteriority $(4,68)$ & $4.513 * *$ & 0.878 \\
Hemi $(1,17)$ & - & 0.000 \\
Anteriority $\times$ Hemi $(4,68)$ & - & 1.219 \\
\hline
\end{tabular}

df: degrees of freedom; Hemi: hemisphere; ${ }^{+} p<0.1 ;{ }^{*} p<0.05 ; * * p<0.01$. 
Table 3. Follow-up analysis of the delay $\times$ anteriority effect, $600-900 \mathrm{~ms}$ from the onset of the pre-delay letter. $F$-values for the effect of delay are given for each anteriority region.

\begin{tabular}{ccc}
\hline Region & Midline, $\mathbf{F}$ & Lateral, $\mathbf{F}$ \\
\hline Frontal & 0.043 & 0.365 \\
Fronto-Central & 0.011 & 1.520 \\
Central & 3.033 & $7.566^{*}$ \\
Central-Parietal & $11.731^{* *}$ & $15.640^{* *}$ \\
Parietal & $14.081^{* *}$ & $21.994^{* * *}$ \\
\hline
\end{tabular}

df: degrees of freedom; ${ }^{*} p<0.05 ;{ }^{* *} p<0.01 ;{ }^{* * *} p<0.001$.

The overall analyses showed a marginal interaction between order and delay at lateral sites (order $\times$ delay, $F(1,17)=4.154, p=0.06$; order $\times$ delay $\times$ anteriority $F(4,68)=3.418, p=0.08$ ). Since we had specifically predicted differences between the random and predictive conditions as a function of the delay, we conducted separate analyses for the delay and no-delay conditions at lateral sites. Results are reported in Table 4. The predictive sequences were more negative than random sequences only in the delay conditions. Results of the follow-up analyses of the effects of order of each anteriority region within the delay condition are given in Table 5. These confirm the observation that the negativity for predictive versus random condition is largest at posterior sites, bilaterally. Means for the lateral sites are given in Figure 3.

Table 4. Delay versus no-delay, 600-900 ms; results from ANOVA (F-values) for lateral sites.

\begin{tabular}{ccc}
\hline Effect $(\mathbf{d f})$ & Delay & No Delay \\
\hline Order $(1,17)$ & $4.908^{*}$ & 0.018 \\
Order $\times$ Anteriority $(4,68)$ & $5.502 *$ & 0.920 \\
Order $\times$ Hemi $(1,17)$ & 0.111 & 0.223 \\
Order $\times$ Anteriority $\times$ Hemi $(4,68)$ & 0.884 & 0.602 \\
Anteriority $(4,68)$ & $3.737 * *$ & 0.273 \\
Hemi $(1,17)$ & 0.098 & 0.094 \\
Anteriority $\times$ Hemi $(4,68)$ & 0.609 & 2.118 \\
\hline
\end{tabular}

df: degrees of freedom; Hemi: hemisphere; ${ }^{*} p<0.05 ;{ }^{* *} p<0.01$.

Table 5. Delay conditions only. Follow up analysis of the order $\times$ anteriority effect, 600-900 ms from the onset of the pre-delay letter at lateral sites. $F$-values for the effect of order are given for each anteriority region.

\begin{tabular}{cc}
\hline Region & Lateral, F \\
\hline Frontal & 0.175 \\
Fronto-Central & 1.694 \\
Central & $5.074 *$ \\
Central-Parietal & $10.978^{* *}$ \\
Parietal & $8.623 * *$ \\
\hline
\end{tabular}

df: degrees of freedom; $* p<0.05 ; * * p<0.01$. 
Figure 3. Mean amplitude $(\mu \mathrm{V})$ in the $600-900-\mathrm{ms}$ window for the lateral regions, collapsed over hemisphere (error bars are standard errors). Blue lines: predictive sequences (Pred); red lines: random sequences; solid lines: delay conditions; dotted lines: conditions without a delay. F: frontal; FC: fronto-central; C: central; CP: central-parietal; P: parietal.

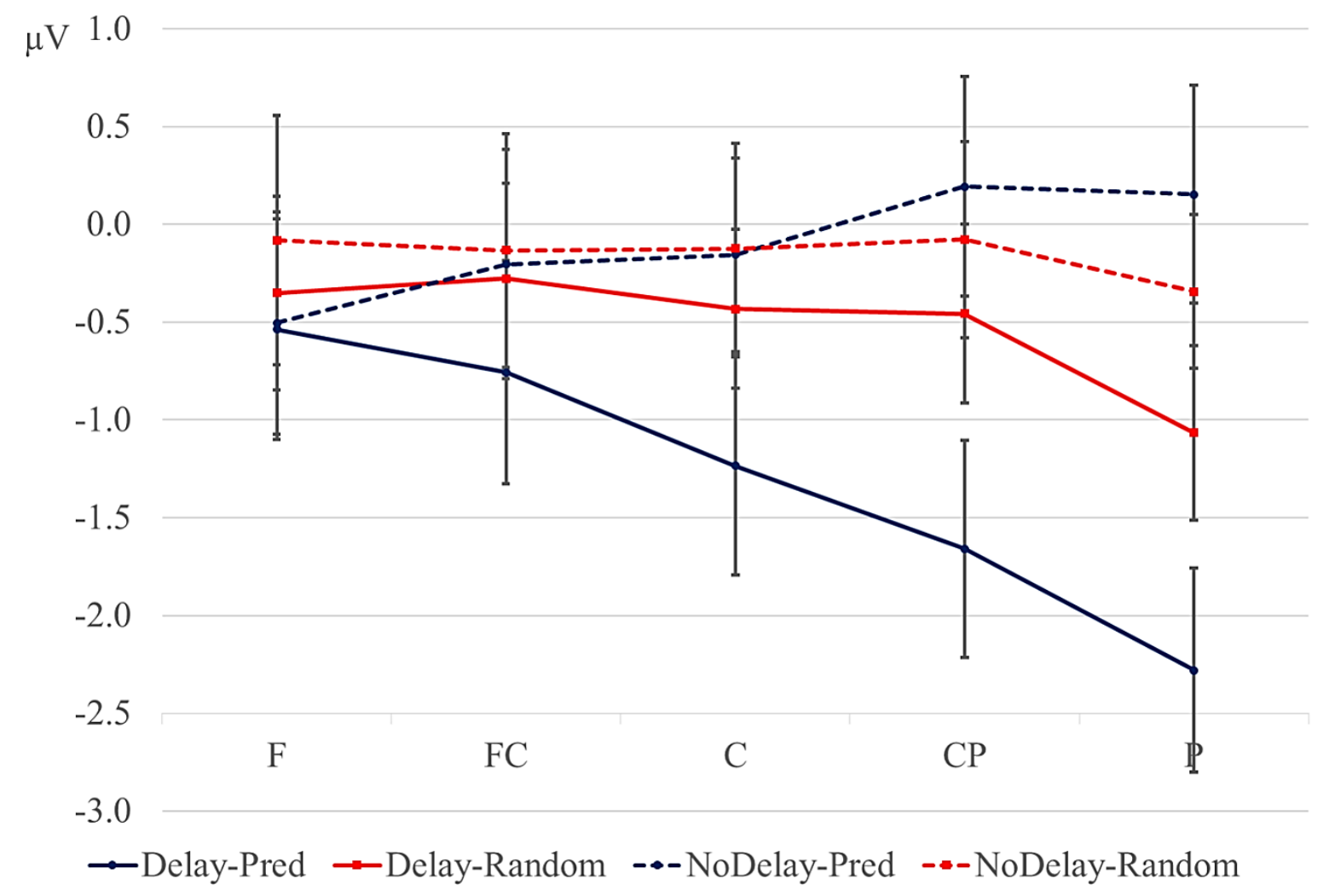

\subsubsection{Post-Delay: 180-280-ms Positivity}

Letters directly following a delay elicited a larger positivity between 180 and $280 \mathrm{~ms}$ at posterior sites compared with letters in similar positions in no-delay trials (see the dotted interval in Figures 1 and 2). This effect was largest over right-hemispheric sites. In addition, the positivity was larger for predictive than for random sequences, but only in the delay conditions. This order effect was broadly distributed (Figure 4). Table 6 gives an overview of the results from the statistical analyses on the mean amplitude of the positivity (that is, 680-780 ms post onset of the preceding letter for the no-delay conditions, 980-1080-ms onset of the pre-delay letter for the delay conditions). Follow-up analyses of the significant interactions between the factors, delay and anteriority, support the observation that the positivity after the delay is largest at posterior sites (Table 7). To assess the delay $\times$ hemisphere interaction, separate analyses were conducted for left and right lateral sites. The effect of delay was significant only over right hemispheric sites (right hemisphere, mean delay effect: $2.25 \mu \mathrm{V} ; F(1,17)=13.627, p<0.01$; left hemisphere, mean delay effect: $0.37 \mu \mathrm{V} ; F(1,17)<1)$.

The interaction of order and delay was followed up by analyses of the delay and no-delay conditions separately; see Table 8 . The positivity was significantly larger for the predictive than the random conditions only in the delay conditions. In contrast to the delay-related negativity (Section 3.2.2), the effect of order did not interact with anteriority (see Figure 4). 
Figure 4. Mean amplitude $(\mu \mathrm{V})$ between 180 and $280 \mathrm{~ms}$ from the onset of the post-delay letter, for the lateral positions, collapsed over hemisphere (error bars are standard errors). Blue lines: predictive sequences (Pred); red lines: random sequences; solid lines: delay conditions; dotted lines: conditions without a delay. F: frontal; FC: fronto-central; C: central; CP: central-parietal; P: parietal.

$$
\mu \mathrm{V} 5.0
$$

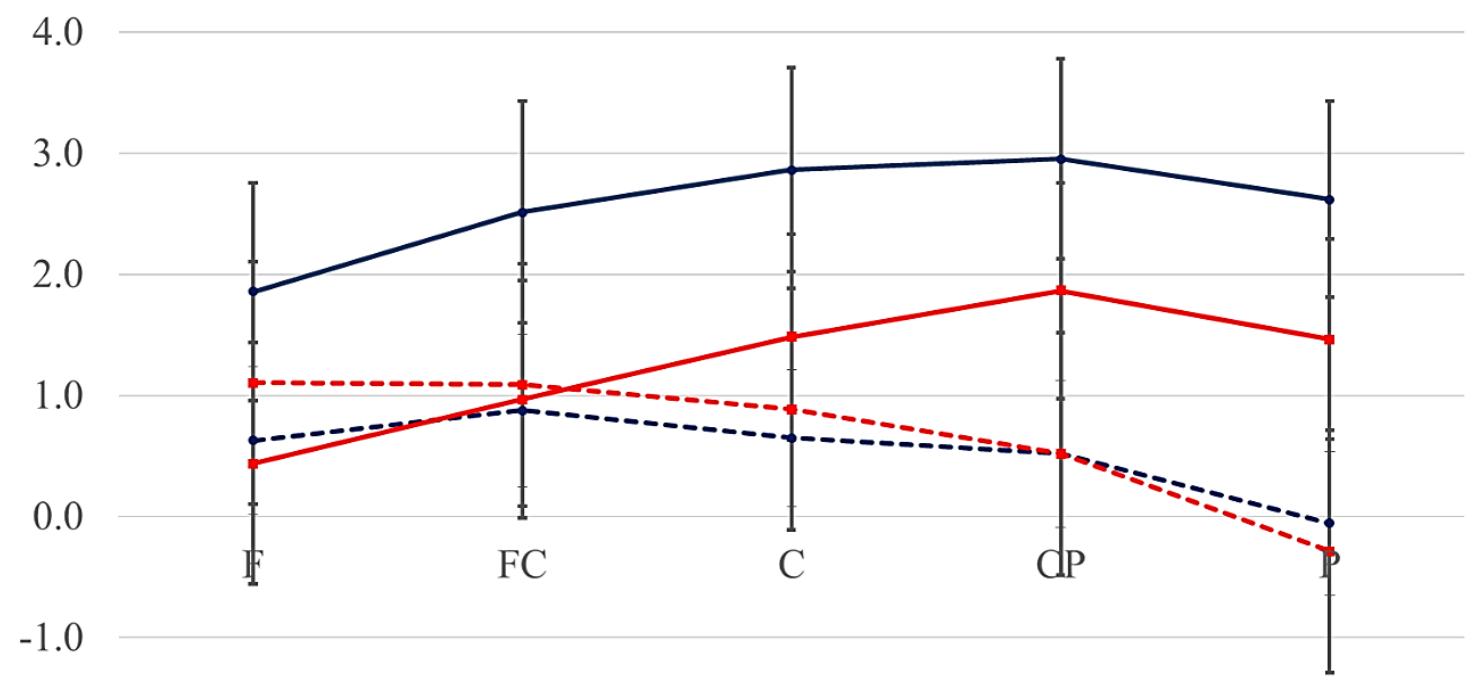

$-2.0$

$\rightarrow-$ NoDelay-Pred $\rightarrow$ Delay-Pred $\quad--$-NoDelay-Random $\rightarrow$ Delay-Random

Table 6. Results from ANOVA ( $F$-values) on the mean amplitudes in the 180-280-ms interval from the onset of the post-delay letter (680-780 ms and 980-1080 ms from the preceding letter in the no delay and delay conditions, respectively).

\begin{tabular}{ccc}
\hline Effect (df) & Midline & Lateral \\
\hline Order $(1,17)$ & 1.974 & $3.069^{+}$ \\
Delay $(1,17)$ & $3.756^{+}$ & $5.114^{*}$ \\
Order $\times$ Anteriority $(4,68)$ & 0.214 & 0.256 \\
Delay $\times$ Anteriority $(4,68)$ & $8.477^{* *}$ & $10.299^{* *}$ \\
Order $\times$ Hemi $(1,17)$ & - & 0.306 \\
Delay $\times$ Hemi $(1,17)$ & - & $33.616^{* * *}$ \\
Order $\times$ Anteriority $\times$ Hemi $(4,68)$ & - & 1.193 \\
Delay $\times$ Anteriority $\times$ Hemi $(4,68)$ & - & 2.169 \\
Order $\times$ Delay $(1,17)$ & $14.765^{* *}$ & $14.022 * *$ \\
Order $\times$ Delay $\times$ Anteriority $(4,68)$ & 0.693 & 0.688 \\
Order $\times$ Delay $\times$ Hemi $(1,17)$ & - & 0.054 \\
Order $\times$ Delay $\times$ Anteriority $\times$ Hemi $(4,68)$ & - & 0.268 \\
Anteriority $(4,68)$ & 1.717 & 0.703 \\
Hemi $(1,17)$ & - & 1.865 \\
Anteriority $\times$ Hemi $(4,68)$ & - & 1.810 \\
\hline
\end{tabular}

df: degrees of freedom; Hemi: hemisphere; ${ }^{+} p<0.1 ; * p<0.05 ; * * p<0.01 ; * * * p<0.001$. 
Table 7. $F$-values for the effect of delay, for each region, 180-280 $\mathrm{ms}$ from the onset of the post-delay letter.

\begin{tabular}{ccc}
\hline Region & Midline df (1,17) & Lateral df (1,17) \\
\hline Frontal & 0.244 & 0.206 \\
Fronto-Central & 1.102 & 1.417 \\
Central & 2.120 & $5.807 *$ \\
Central-Parietal & $6.526^{*}$ & $9.152 * *$ \\
Parietal & $12.400^{* *}$ & $12.075^{* *}$ \\
\hline
\end{tabular}

df: degrees of freedom; $* p<0.05 ; * * p<0.01$.

Table 8. Results for follow-up ANOVA ( $F$-values), 180-280 ms from the post-delay letter, separately for delay and no-delay conditions.

\begin{tabular}{ccccc}
\hline \multirow{2}{*}{ Effect (df) } & \multicolumn{2}{c}{ Delay $(\mathbf{9 8 0}-\mathbf{1 0 8 0} \mathbf{~ m s})$} & \multicolumn{2}{c}{ No Delay $(\mathbf{6 8 0}-\mathbf{7 8 0} \mathbf{~ m s})$} \\
& Midline & Lateral & Midline & Lateral \\
\hline Order $(1,17)$ & $9.032 * *$ & $10.581 * *$ & 1.242 & 0.141 \\
Order $\times$ Anteriority $(4,68)$ & 0.967 & 0.732 & 0.309 & 0.483 \\
Order $\times$ Hemi $(1,17)$ & - & 0.016 & - & 0.302 \\
Order $\times$ Anteriority $\times$ Hemi $(4,68)$ & - & 0.667 & - & 0.919 \\
Anteriority $(4,68)$ & 2.318 & 1.515 & $4.029^{*}$ & 3.374 \\
Hemi $(1,17)$ & - & $9.862 * *$ & - & 1.265 \\
Anteriority $\times$ Hemi $(4,68)$ & - & 2.069 & - & 1.435 \\
\hline
\end{tabular}

df: degrees of freedom; $* * p<0.01$.

\subsubsection{Other Effects}

As can be seen in Figure 1, the 180-280-ms positivity overlapped with a longer-lasting frontal negativity for the random versus predictive delay conditions. This observation was supported by a significant interaction between anteriority and order for the 1200-1400 ms interval from the onset of the pre-delay letter, that is $400-600 \mathrm{~ms}$ from the onset of the post-delay letter (midline: $F(4,68)=5.603$, $p<0.05$; lateral: $F(4,68)=7.356, p<0.01)$. Follow-up analyses confirmed that the effect of order was present only at frontal sites (frontal lateral regions: $F(1,17)=4.561, p<0.05$; other regions, $p$ s $>0.1)$. Although the effect seemed largest over left frontal and central sites, the three-way interaction between order, anteriority and hemisphere did not reach significance $(F(4,68)=1.364, p=0.26)$.

Finally, visual inspection of the results for the no-delay conditions in Figure 2 suggested that ERPs for the random sequences were more negative than for the predictive conditions at left posterior sites between 800 and $1000 \mathrm{~ms}$ and more positive at frontal sites between 1000 and $1200 \mathrm{~ms}$ after the onset of the pre-delay letter equivalent. However, analyses for these two intervals in the no-delay conditions yielded no significant effects involving the factor order (order $\times$ hemisphere $800-1,000 \mathrm{~ms}, F(1,17)=3.197$; $p=0.09$; other effects, $p \mathrm{~s}>0.5$ ).

\subsection{ERPs: Effects of Predictive vs. Random Sequences (Time Locked to First Letter)}

In order to further explore differences between random and predictive sequences, we investigated ERPs to the first few letters of each trial. At the first letter of each trial, participants did not know whether 
the sequence was random or predictive. Only the second letter was informative in this respect. Differences in ERPs at the second letter were therefore not confounded by pre-existing differences between the predictive and random conditions. Figure 5 displays the ERPs starting from the presentation of the first letter in the sequence. The $\mathrm{P} 2$ to the second letter was larger for the predictive than for the random conditions, especially at anterior sites (Figure 6). Results from the ANOVA are given in Table 9. In the window spanning the $\mathrm{P} 2$ at the second letter (680-780 ms from the onset of the first letter), a marginal interaction between order and anteriority was found in the analysis of the lateral sites $(p=0.051)$. Follow-up analyses for each anteriority region (Table 10) supported the observation that the increased positivity for predictive versus random sequences was largest at frontal and fronto-central sites.

Figure 5. ERPs time-locked to the onset of the first letter in the sequence. ERPs are baselined to $100 \mathrm{~ms}$ preceding the onset of the first letter. Letters are presented for $300 \mathrm{~ms}$, starting at $0 \mathrm{~ms}, 500 \mathrm{~ms}$ and $1000 \mathrm{~ms}$. Blue line: predictive (alphabetic) condition; red line: random condition. Dotted lines: window for the analysis of the P2; shaded intervals: windows used for the analysis of the negativity (dark bar: $800-1000 \mathrm{~ms}$; light bar: 1300-1500 ms).
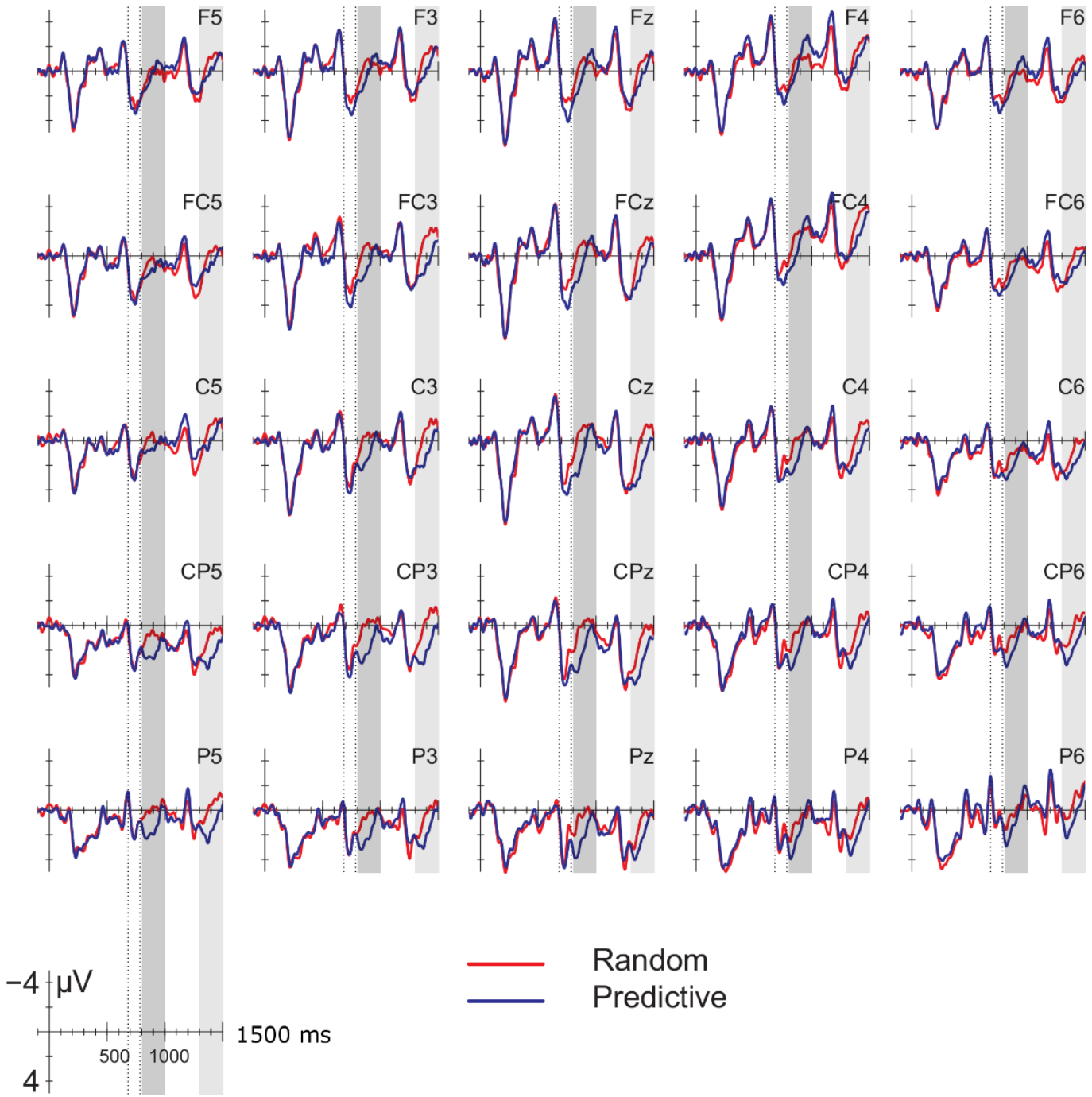

Random

Predictive 
Figure 6. Mean amplitude $(\mu \mathrm{V})$ between 180 and $280 \mathrm{~ms}$ from the onset of the second letter in the trial, for the lateral positions, collapsed over hemisphere and delay (error bars are standard errors). Blue lines: predictive sequences; red lines: random sequences. F: frontal; FC: fronto-central; C: central; CP: central-parietal; P: parietal.

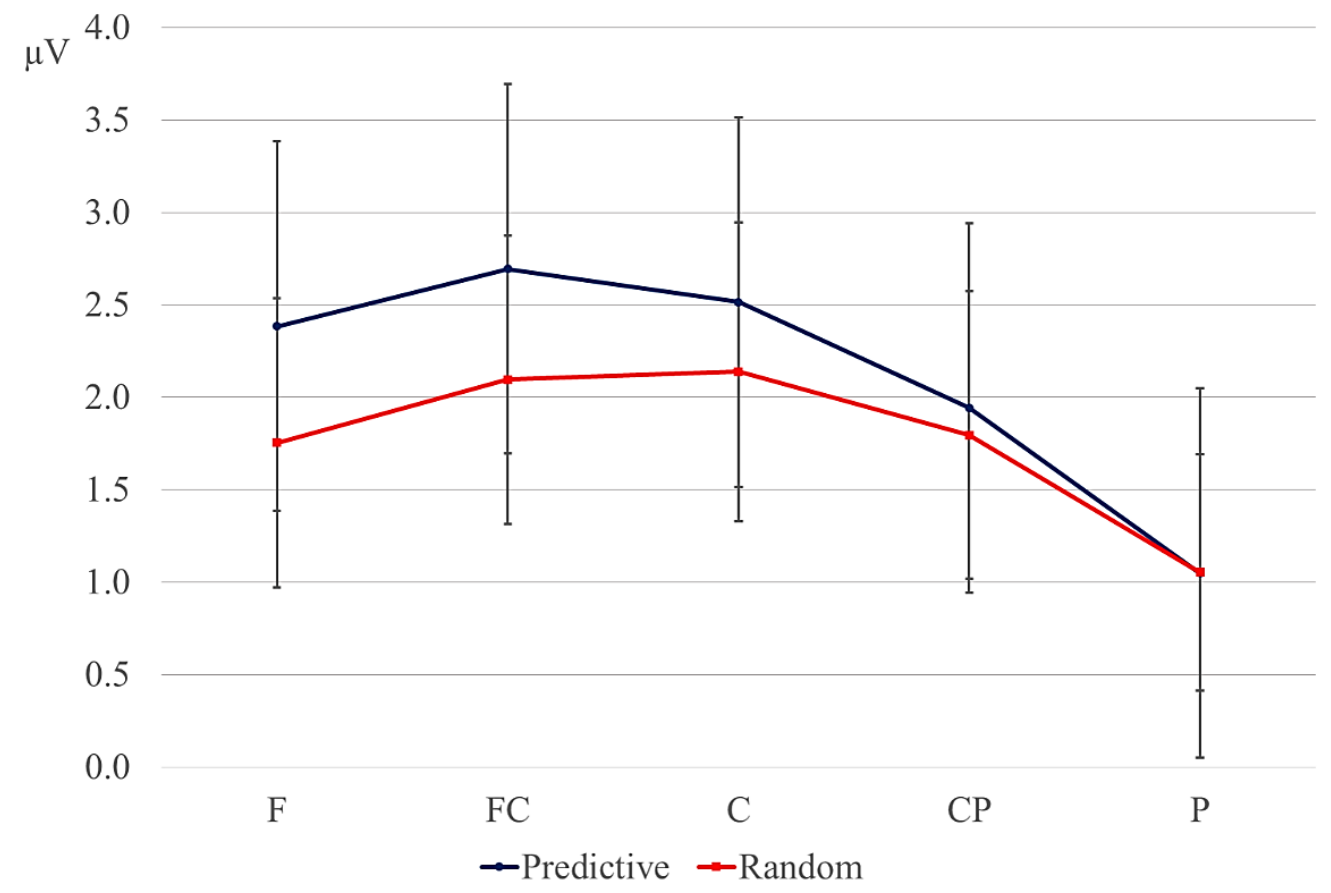

Table 9. First letter, results from ANOVA (F-values) for the 680-780-ms (P2), 800-1000-ms and 1300-1500-ms (negativity) intervals.

\begin{tabular}{ccccccc}
\hline \multirow{2}{*}{ Effect (df) } & \multicolumn{2}{c}{$\mathbf{6 8 0 - 7 8 0 ~} \mathbf{~ m s}$} & \multicolumn{2}{c}{$\mathbf{8 0 0 - 1 0 0 0 ~} \mathbf{~ m s}$} & \multicolumn{2}{c}{$\mathbf{1 3 0 0 - 1 5 0 0 ~ m s}$} \\
\cline { 2 - 7 } & Midline & Lateral & Midline & Lateral & Midline & Lateral \\
\hline Order $(1,17)$ & $5.745^{*}$ & 1.703 & $10.330^{* *}$ & $6.733^{*}$ & $6.300^{*}$ & $6.246^{*}$ \\
Order $\times$ Anteriority $(4,68)$ & 1.174 & $3.685^{+}$ & $4.058^{*}$ & $5.890^{* *}$ & 1.953 & 2.598 \\
Order $\times$ Hemi $(1,17)$ & - & 0.017 & - & 1.835 & - & 0.338 \\
Order $\times$ Anteriority $\times$ Hemi $(4,68)$ & - & 0.409 & - & 1.577 & - & 0.637 \\
Anteriority $(4,68)$ & 1.028 & $4.793^{*}$ & $3.142^{*}$ & 1.961 & $2.916^{+}$ & $2.543^{+}$ \\
Hemi $(1,17)$ & - & $4.621^{*}$ & - & 0.686 & - & 0.054 \\
Anteriority $\times$ Hemi $(4,68)$ & - & 0.303 & - & 0.675 & - & 0.518 \\
\hline
\end{tabular}

df: degrees of freedom; Hemi: hemisphere; ${ }^{+} p<0.1{ }^{*} p<0.05 ; * * p<0.01$.

Table 10. First letter, $F$-values for the effect of order, for each anteriority region, 680-780 ms (P2) and 800-1000 ms (negativity).

\begin{tabular}{cccc}
\hline \multirow{2}{*}{ Region } & $\mathbf{6 8 0 - 7 8 0 ~} \mathbf{~ m s}$ & \multicolumn{2}{c}{$\mathbf{8 0 0}-\mathbf{1 0 0 0} \mathbf{~ m s}$} \\
\cline { 2 - 4 } & Lateral, $\mathbf{F}$ & Midline, $\mathbf{T}$ & Lateral, $\mathbf{F}$ \\
\hline Frontal & $3.831^{+}$ & 0.866 & 0.079 \\
Fronto-Central & $4.947^{*}$ & $1.961^{+}$ & $3.676^{+}$ \\
Central & 1.887 & $3.205^{* *}$ & $6.459^{*}$ \\
Central-Parietal & 0.212 & $3.942^{* *}$ & $9.414^{* *}$ \\
Parietal & 0.000 & $3.626^{* *}$ & $11.055^{* *}$ \\
\hline
\end{tabular}

df: degrees of freedom; ${ }^{+} p<0.1 ;{ }^{*} p<0.05 ; * * p<0.01$. 
Second, resembling the pattern observed for the interval preceding the pre-delay letter (Section 3.2.1), the random and predictive conditions differed in that the ERPs at posterior sites were more negative for the random than for the predictive condition between the offset of the second letter $(800 \mathrm{~ms})$ and the onset of the next letter $(1000 \mathrm{~ms})$. This negativity was repeated, although less robustly, at the next letter in the sequence (1300-1500 ms). Results from the ANOVA are given in Table 9. In the 800-1000-ms window, a significant interaction was found between order and anteriority. Separate analyses for each of the anteriority regions (Table 10) confirmed that the negativity for random versus predictive sequences had a posterior maximum. Between 1300 and $1500 \mathrm{~ms}$, only the effect of order was significant.

\section{Discussion}

The aim of the present study was to probe implicit predictive processes during the processing of alphabetical versus random letter sequences using a delay paradigm without feedback. We occasionally lengthened the ISI between two letters by $300 \mathrm{~ms}$. This manipulation allowed us to observe whether: (1) the ERPs elicited during this delay period were sensitive to the difference in predictability of the upcoming stimulus; and (2) the delay and predictability were affected the pre-activation and processing of the next letter. During the delay period, the predictive sequences elicited a larger posterior negativity than the random sequences. In contrast, random versus predictive conditions showed a 300-500-ms negativity in some positions without or before a delay. Second, letters following the delay showed a larger posterior positivity between 180 and $280 \mathrm{~ms}$ after the onset of the post-delay letter than letters in comparable positions in the no-delay sequences. The 180-280-ms positivity was larger for predictive than for random letters following a delay. No effect of predictability on the positivity was seen in the no-delay comparisons, although we did observe such a modulation at the second letter from the beginning of each trial. We will discuss these effects in turn.

\subsection{Delay-Related Negativity}

The larger negativity observed during the delay for the predictive versus random sequences can be interpreted in several ways. First, it can be an SPN, indexing the expectation of the upcoming letter; second, the increased negativity can reflect a violation of the expected presentation of the next letter; third, the negativity may be related to task-effects. We will discuss these interpretations in turn.

SPNs have typically been observed when the participant expects a stimulus that is informative, either because it is a response probe, instructional probe, feedback, a reward or an affective stimulus [13]. In our study, the letter stimuli in the trials are not informative in the sense of being instructions, probes or feedback, but we do find a larger negativity in the interval preceding highly predictive letters compared with random letters. A larger SPN for predictive sequences is at odds with previous observations that the SPN is smaller preceding highly predictable compared with less predictable stimuli [15-17], but see [18]. Even though the letters in our alphabetical sequences were $100 \%$ predictable and, strictly speaking, uninformative, participants could have engaged themselves by internally generating the next letter during the delay interval in the predictive trials; this as opposed to the random sequences, in which a correct prediction of the upcoming letter was not very likely. The next letter in the predictive sequences then served as implicit feedback that the participant used to 
check the internally generated prediction. The SPN then reflects the expectation of this implicit feedback stimulus. The support for this interpretation is that the 180-280-ms positivity component after the delay was larger for the predicted than for the random letters (see below). This suggests that the form and/or concept of the upcoming letter was anticipated and pre-activated during the delay period. A potential problem with interpreting the delay-related negativity as a reflection of the anticipation of the identity of the next letter is that no such negativity was found in the no-delay predictive conditions, even though participants would be likely to pre-activate the next letter in the no-delay conditions, as well. Instead, the ERPs in the random no-delay conditions were more negative at posterior sites relative to the predictive no-delay conditions, especially at earlier positions in the trial. Given its scalp distribution and timing, we speculatively interpret the posterior negativity for the random versus predictive conditions as an N400 effect. Since the letters in the predictive, alphabetic sequences were highly associated, the larger N400 effect may index weaker priming between the letters in the random sequences. If the negativity we observed for the predictive sequences during the delay period is an SPN, one must therefore assume that the expectation of the particular identity of the next letter is boosted by the delay. Without the delay, the expectation is not strong enough to overcome priming-related effects.

An alternative interpretation of the delay-related negativity is that it reflects an omission error [19-21,36]. Given the fixed presentation rate of the stimuli preceding the delay in our study, the participant may have anticipated the presentation of the next letter at a fixed point in time. This expectation is violated by the delay, giving rise to the negativity. Assuming that the expectation for the presentation of the following letter was stronger in the predictive than random conditions, the increase in negativity in the former condition can be accounted for [22,23]. The posterior distribution of the negativity matches the posterior effects found in some omission-studies using visual stimuli $[24,25]$. Somewhat problematic for interpreting the negativity in terms of an omission error is that $50 \%$ of the trials in the current study contained a delay after the fifth or later letter in the sequence. The delay could therefore have been anticipated to some extent by the participants, making it somewhat unlikely that the negativity reflects an omission error. Furthermore, one would expect both the random and predictive conditions to elicit an omission-related negativity, as in both cases, the presentation of the letters is rhythmic and the delay violates this temporal regularity. Although a better control condition is needed to evaluate this prediction, the ERPs to the random delay condition seem to hardly deviate from the baseline (Figures 2 and 3).

Other interpretations of the delay-related difference between random and predictive sequences cannot be excluded. One confounding factor may have been the use of a letter verification task at the end of each trial, which may have led to processing differences between the random and predictive conditions. In the debriefing after the study, some participants mentioned they encoded only the first and last letters of a predictive sequence. At the probe, they would reconstruct the sequence in between the first and last letters to match the probe. In the random sequences, on the other hand, they reported encoding each letter as it appeared. Results from the letter verification task match these observations: responses to the predictive sequences were more accurate, but slower than responses to the random sequences. The difference in ERPs between the predictive and random sequences observed during the delay period may therefore also be related to a deeper encoding for the random sequences. Somewhat problematic for this interpretation, however, is that deeper encoding is associated with a larger positivity [37,38]. Although 
the random conditions were relatively more positive than the predictive conditions during the delay, the random conditions lack a clear positive going component.

In order to distinguish among the interpretations of the negativity, follow-up studies are needed in which the task demands, the degree of predictability of the next stimulus and the predictability of the delay are varied. If the negativity is indeed sensitive to the predictability of the next stimulus, the negativity is expected to vary as a function of predictability [15] (but see [39]), but not as a function of the predictability of the delay. If, on the other hand, the negativity is an omission error, it will not occur when a delay is predicted to occur. If the negativity we observed is due to task demands, we should no longer observe the amplitude difference between predictive and random sequences with a different task. Although more research is needed, the current finding that the delay-related negativity was sensitive to the difference in predicted versus random sequences supports the view that the negativity reflects differences in expectation and that similar delay-paradigms can be used to further study predictive processing.

\subsection{0-280-ms Positivity: P3b and P2}

We observed two modulations of the 180-280-ms positivity at the letter following the potential delay. First, a larger posterior positivity was elicited after a delay compared with letters in similar positions not following a delay. Given its posterior distribution, we identify this positivity as the P3b, which is typically seen for task-related targets and is larger when the participant devotes more resources to the task $[40,41]$. Under this interpretation, the delay in our paradigm may have focused the participants' attention on the upcoming stimulus letter. Alternatively, or in addition, the stronger positivity after the delay may be related to refractory effects: early visual and auditory components have been found to be larger in amplitude with longer ISIs [42-45].

Second, we found that the 180-280-ms positivity was larger for the predictive than random sequences, but only after a delay. This effect of order could be seen over the entire scalp. One interpretation is that the effect is a modulation of the P2 component. The P2 has been associated with the ease of the detection of particular sensory features [26] and with the matching of the actual visual input with an expected form [27]. Given this interpretation, our data suggests that the delay period enabled our participants to generate an expectation of the form of the next letter in the predictive sequences. This pre-activated the visual system and led to a larger P2 for the predictive versus non-predictive sequences. Similarly, the 180-280-ms positivity was larger for the predictive than for the random sequences at the second position in each trial, although this effect was more frontally distributed. The increased positivity for the predictive conditions may have reflected the pre-activation of the next letter in the alphabet upon seeing the first letter in the trial. The absence of the P2 effect at later (no-delay) positions suggests that the effects of expectation on visual processing are strongest when there is less adaption of the P2 to the visual stimuli. We are somewhat cautious in interpreting the difference observed between the random and predictive prediction after the delay as a $\mathrm{P} 2$, however, since the difference between the conditions may have been caused or enhanced by the anterior negativity for the random conditions observed after the delay. This continued anterior difference between predictive and random conditions can be interpreted as a memory related component for the random condition [46]. 
As mentioned previously, the random conditions may have required more encoding. This encoding may have been more taxing directly following a potentially distracting delay.

Note that even at posterior positions, where the superimposed negative effect was least apparent, the positivity following the delay was larger for the predictive than the random conditions. This suggests that also the P3b is sensitive to predictability of the stimulus [31-33]. This supports the view that the delay leads to a stronger pre-activation of the upcoming stimulus and, therefore, a more apparent match of expectancy when the actual stimulus appears, leading to a larger P3b. Regardless of whether the positivity is interpreted as a P2 or P3b, our results lend support to the view that an expected form or concept is pre-activated during the delay and subsequently matched against the actual stimulus.

\section{Conclusions}

Most previous studies on anticipation in sequential processing have used paradigms in which the participant learns to associate a particular stimulus with a particular outcome and in which explicit feedback is provided. We have shown that an anticipation-related negativity can be elicited by stimuli in sequences that are based on long-term associations and in which no feedback is provided. In addition, our results suggests that some features of the anticipated stimulus are pre-activated during the delay period.

The present paradigm can be applied to the study of predictive processing during sentence comprehension. In contrast to the delay paradigms used in non-language studies, most studies on anticipation in language processing have focused on ERPs elicited by words that violate or meet context-based predictions [47-52]. ERPs at these word positions also reflect integration and revision processes, which makes it hard to distinguish predictive processing from other processes. The delay paradigm can be easily applied to sentence processing [17]: the next word in a sentence can be occasionally delayed, enabling the tracking of anticipatory processes separately from integration, while still encouraging readers to process the materials following the delay, while avoiding anomalies inherent to omission [22] and violation paradigms [47-52]. We are currently applying the delay paradigm to sentence processing in which the cloze probability of the post-delay word is manipulated [53]. In addition, by manipulating the length of the delay, one can yield a better view of the time needed for certain predictions to be generated and whether there are differences in populations in this respect, such as between second-language learners with different levels of proficiency. In sum, the delay paradigm may be a fruitful method to determine the nature and time course of anticipations during the processing of sequential stimuli, be it linguistic or otherwise.

\section{Acknowledgments}

The authors would like to thank George Collins for his help collecting data and Jingchen Xu for assisting with the figures. 


\section{Author Contributions}

Edith Kaan designed the study, performed the ERP analysis and wrote the manuscript. Evan Carlisle prepared and conducted the study, preprocessed the ERP data, analyzed the behavioral data and contributed to parts of the method and results sections of the paper.

\section{Conflicts of Interest}

The authors declare no conflict of interest.

\section{References}

1. Bar, M. The proactive brain: Memory for predictions. Philos. Trans. R. Soc. Lond. B Biol. Sci. 2009, 364, 1235-1243.

2. Clark, A. Whatever next? Predictive brains, situated agents, and the future of cognitive science. Behav. Brain Sci. 2013, 36, 181-204.

3. Chang, F.; Dell, G.S.; Bock, K. Becoming syntactic. Psychol. Rev. 2006, 113, 234-272.

4. Altmann, G.T.M.; Mirkovic, J. Incrementality and prediction in human sentence processing. Cogn. Sci. 2009, 33, 583-609.

5. Jaeger, T.F.; Snider, N.E. Alignment as a consequence of expectation adaptation: Syntactic priming is affected by the prime's prediction error given both prior and recent experience. Cognition 2013, 127, 57-83.

6. Kutas, M.; DeLong, K.A.; Smith, N.J. A look around at what lies ahead: Prediction and predictability in language processing. In Predictions in the Brain: Using Our Past to Generate A Future; Bar, M., Ed.; Oxford University Press: New York, NY, USA, 2011; pp. 190-207.

7. Levy, R. Expectation-based syntactic comprehension. Cognition 2008, 106, 1126-1177.

8. Pickering, M.J.; Garrod, S. An integrated theory of language production and comprehension. Behav. Brain Sci. 2013, 36, 329-347.

9. Van Petten, C.; Luka, B.J. Prediction during language comprehension: Benefits, costs, and ERP components. Int. J. Psychophysiol. 2012, 83, 176-190.

10. MacDonald, M.C. How language production shapes language form and comprehension. Front. Psychol. 2013, 4, 226; doi:10.3389/fpsyg.2013.00226.

11. Damen, E.J.P.; Brunia, C.H.M. Changes in heart rate and slow brain potentials related to motor preparation and stimulus anticipation in a time estimation task. Psychophysiology 1987, 24, 700-713.

12. Böcker, K.B.E.; Van Boxtel, G.J.M. Stimulus-preceding negativity: A class of anticipatory slow potentials. In Brain and Behavior: Past, Present, and Future; Böcker, K.B.E., van Boxtel, G.J.M., Eds.; Tilburg University Press: Tilburg, The Netherlands, 1997; pp. 105-116.

13. Van Boxtel, G.J.M.; Böcker, K.B.E. Cortical measures of anticipation. J. Psychophysiol. 2004, 18, 61-76.

14. Damen, E.J.P.; Brunia, C.H.M. Is a stimulus conveying task-relevant information a sufficient condition to elicit a stimulus-preceding negativity? Psychophysiology 1994, 31, 129-139.

15. Catena, A.; Perales, J.C.; Megías, A.; Cándido, A.; Jara, E.; Maldonado, A. The brain network of expectancy and uncertainty processing. PLoS One 2012, 7, e40252; doi:10.1371/journal.pone.0040252. 
16. Morís, J.; Luque, D.; Rodríguez-Fornells, A. Learning-induced modulations of the stimulus-preceding negativity. Psychophysiology 2013, 50, 931-939.

17. Besson, M.; Faita, F.; Czternasty, C.; Kutas, M. What's in a pause: Event-related potential analysis of temporal disruptions in written and spoken sentences. Biol. Psychol. 1997, 46, 3-23.

18. Chwilla, D.J.; Brunia, C.H.M. Event-related potential to different feedback stimuli. Psychophysiology 1991, 28, 123-132.

19. Tervaniemi, M.; Saarinen, J.; Paavilainen, P.; Danilova, N.; Näätänen, R. Temporal integration of auditory information in sensory memory as reflected by the mismatch negativity. Biol. Psychol. 1994, 38, 157-167.

20. Yabe, H.; Tervaniemi, M.; Reinikainen, K.; Näätänen, R. Temporal window of integration revealed by MMN to sound omission. Neuroreport 1997, 8, 1971-1974.

21. Pihko, E.; Leppasaari, T.; Ulla Richardson, P.L.; Lyytinen, H. Auditory event-related potentials (ERP) reflect temporal changes in speech stimuli. Neuroreport 1997, 8, 911-914.

22. Bendixen, A.; Scharinger, M.; Strauß, A.; Obleser, J. Prediction in the service of comprehension: Modulated early brain responses to omitted speech segments. Cortex 2014, 53, 9-26.

23. Mattys, S.L.; Pleydell-Pearce, C.W.; Melhorn, J.F.; Whitecross, S.E. Detecting silent pauses in speech: A new tool for measuring on-line lexical and semantic processing. Psychol. Sci. 2005, 16, 958-964.

24. Renault, B.; Lesevre, N. Topographical study of the emitted potential obtained after the omission of an expected visual stimulus. In Multidisciplinary Perspectives in Event-Related Brain Potential Research; Otto, D., Ed.; Government Printing Office: Washington, DC, USA, 1978; pp. 202-208.

25. Simson, R.; Vaughan, H.G.; Ritter, W. The scalp topography of potentials associated with missing visual or auditory stimuli. Electroencephalogr. Clin. Neurophysiol. 1976, 40, 33-42.

26. Luck, S.J.; Hillyard, S.A. Electrophysiological correlates of feature analysis during visual search. Psychophysiology 1994, 31, 291-308.

27. Evans, K.M.; Federmeier, K.D. Left and right memory revisited: Electrophysiological investigations of hemispheric asymmetries at retrieval. Neuropsychologia 2009, 47, 303-313.

28. Misra, M.; Holcomb, P.J. Event-related potential indices of masked repetition priming. Psychophysiology 2003, 40, 115-130.

29. Federmeier, K.D.; Mai, H.; Kutas, M. Both sides get the point: Hemispheric sensitivities to sentential constraint. Mem. Cogn. 2005, 33, 871-886.

30. Molinaro, N.; Barraza, P.; Carreiras, M. Long-range neural synchronization supports fast and efficient reading: EEG correlates of processing expected words in sentences. NeuroImage 2013, 72, $120-132$.

31. Molinaro, N.; Carreiras, M. Electrophysiological evidence of interaction between contextual expectation and semantic integration during the processing of collocations. Biol. Psychol. 2010, 83, 176-190.

32. Vespignani, F.; Canal, P.; Molinaro, N.; Fonda, S.; Cacciari, C. Predictive mechanisms in idiom comprehension. J. Cogn. Neurosci. 2009, 22, 1682-1700.

33. Roehm, D.; Bornkessel-Schlesewsky, I.; Rösler, F.; Schlesewsky, M. To predict or not to predict: Influences of task and strategy on the processing of semantic relations. J. Cogn. Neurosci. 2007, 19, $1259-1274$. 
34. Greenhouse, S.W.; Geisser, S. On methods in the analysis of profile data. Psychometrika 1959, 24, 95-112.

35. Leys, C.; Ley, C.; Klein, O.; Bernard, P.; Licata, L. Detecting outliers: Do not use standard deviation around the mean, use absolute deviation around the median. J. Exp. Soc. Psychol. 2013, 49, 764-766.

36. SanMiguel, I.; Widmann, A.; Bendixen, A.; Trujillo-Barreto, N.; Schröger, E. Hearing silences: Human auditory processing relies on preactivation of sound-specific brain activity patterns. J. Neurosci. 2013, 33, 8633-8639.

37. Friedman, D. ERPs during continuous recognition memory for words. Biol. Psychol. 1990, 30, 61-87.

38. Paller, K.A.; Kutas, M.; Mayes, A.R. Neural correlates of encoding in an incidental learning paradigm. Electroencephalogr. Clin. Neurophysiol. 1987, 67, 360-371.

39. Stadler, W.; Klimesch, W.; Pouthas, V.; Ragot, R. Differential effects of the stimulus sequence on CNV and P300. Brain Res. 2006, 1123, 157-167.

40. Isreal, J.B.; Chesney, G.L.; Wickens, C.D.; Donchin, E. P300 and tracking difficulty: Evidence for multiple resources in dual-task performance. Psychophysiology 1980, 17, 259-273.

41. Donchin, E.; Ritter, W.; McCallum, W.G. Cognitive psychophysiology: The endogenous components of the ERP. In Brain Event Related Potentials in Man; Callaway, E., Teuting, P., Koslow, S., Eds.; Academic Press: New York, NY, USA, 1978; pp. 349-411.

42. Knight, R.T.; Hillyard, S.A.; Woods, D.L.; Neville, H.J. The effects of frontal and temporal-parietal lesions on the auditory evoked potential in man. Electroencephalogr. Clini. Neurophysiol. 1980, 50, 112-124.

43. Holcomb, P.; Neville, H. Natural speech processing: An analysis using event-related brain potentials. Psychobiology 1991, 19, 286-300.

44. Gastaut, H.; Gastaut, Y.; Roger, A.; Carriol, J.; Naquet, R. Étude électrographique du cycle d'excitabilité cortical. Electroencephalogr. Clin. Neurophysiol. 1951, 3, 401-428.

45. Lehtonen, J.B. Functional differentiation between late components of visual evoked potentials recorded at occiput and vertex: Effect of stimulus interval and contour. Electroencephalogr. Clin. Neurophysiol. 1973, 35, 75-82.

46. Friedman, D.; Johnson, R.J. Event-related potential (ERP) studies of memory encoding and retrieval: A selective review. Microsc. Res. Tech. 2000, 51, 6-28.

47. Van Berkum, J.J.A.; Brown, C.M.; Zwitserlood, P.; Kooijman, V.; Hagoort, P. Anticipating upcoming words in discourse: Evidence from ERPs and reading times. J. Exp. Psychol. Learn. Mem. Cogn. 2005, 31, 443-467.

48. Wicha, N.Y.Y.; Moreno, E.M.; Kutas, M. Expecting gender: An event related brain potential study on the role of grammatical gender in comprehending a line drawing within a written sentence in Spanish. Cortex 2003, 39, 483-508.

49. Wicha, N.Y.Y.; Moreno, E.M.; Kutas, M. Anticipating words and their gender: An event-related brain potential study of semantic integration, gender expectancy, and gender agreement in spanish sentence reading. J. Cogn. Neurosci. 2004, 16, 1272-1288.

50. DeLong, K.A.; Urbach, T.P.; Kutas, M. Probabilistic word pre-activation during language comprehension inferred from electrical brain activity. Nat. Neurosci. 2005, 8, 1117-1121. 
51. Federmeier, K.D.; Wlotko, E.W.; De Ochoa-Dewald, E.; Kutas, M. Multiple effects of sentential constraint on word processing. Brain Res. 2007, 1146, 75-84.

52. Laszlo, S.; Federmeier, K.D. A beautiful day in the neighborhood: An event-related potential study of lexical relationships and prediction in context. J. Mem. Lang. 2009, 61, 326-338.

53. Kaan, E.; Carlisle, E.; Collins, G.B.; Feroce, N.; Reyes, A. ERP indices of predictive processing. In Proceedings of the CUNY Conference on Human Sentence Processing, Columbus, OH, USA, 13-15 March 2014.

(C) 2014 by the authors; licensee MDPI, Basel, Switzerland. This article is an open access article distributed under the terms and conditions of the Creative Commons Attribution license (http://creativecommons.org/licenses/by/4.0/). 